\title{
NOUVELLE
}

\section{Don't worry, be healthy! Les effets délétères du stress psychique sur la réponse aux thérapies anti-tumorales}

Nassim Salem ${ }^{1}$, Pierre-Emmanuel Foy ${ }^{2}$

\author{
${ }^{1}$ Master 2 Immunologie Translationnelle et Biothérapies, \\ Mention BMC, Sorbonne Université, Paris, France. \\ ${ }^{2}$ Master 2 Immunologie Intégrative et Systémique, \\ Mention BMC, Sorbonne Université, Paris, France. \\ salemnassim96@gmail.com \\ p.e.foy@hotmail.fr
}

Le système immunitaire, un élément clé de la réponse aux thérapies anti-cancéreuses Depuis quelques années, cibler le système immunitaire afin de le rendre plus efficace contre les tumeurs est une approche innovante et particulièrement prometteuse en thérapie anti-cancéreuse. Dès le début du $\mathrm{xx}$ siècle, les travaux de Coley consistant à utiliser des extraits bactériens chez des patients atteints de sarcome afin de stimuler leur réponse anti-tumorale, ont mis en évidence le rôle clé de l'immunité adaptative dans le contrôle de la croissance tumorale. Pour autant, il faut attendre les travaux de J. Allison et T. Honjo sur les points de contrôle immunitaire ou immune checkpoints pour voir apparaître une nouvelle classe d'immunothérapie ciblant directement les cellules immunitaires afin de réactiver l'immunité anti-tumorale. Ces deux chercheurs ont tous les deux été récompensés par le prix Nobel de physiologie ou médecine en 2018 pour leur travaux sur les molécules CTLA-4 (cloné à Marseille en 1987) [1] et PD-1, les deux immune checkpoints les plus ciblés en immunothérapie des cancers [11, 12] $(\rightarrow)$. Malgré une

$\rightarrow$ Voir Les Repères (Nobel 2018) de J.L. Teillaud et W.H. Fridman et J.L. Teillaud, $\mathrm{m} / \mathrm{s}$ $n^{\circ} 4$, avril 2019, pages $365 \mathrm{t} 367$ efficacité chez des patients non répondeurs aux thérapies conventionnelles dans des cancers de mauvais pronostic comme le mélanome métastatique, moins de $20 \%$ des patients répondent généralement à ces immunothérapies. De nombreux défis restent donc à relever afin d'augmenter l'efficacité de cette stratégie thérapeutique [2].

Récemment, des études ont montré que l'efficacité de certains traitements conventionnels tumoricides (chimiothérapie, radiothérapie) repose aussi en partie sur l'induction d'une réponse immunitaire anti-tumorale. En effet, ces traitements, en induisant la destruction des cellules tumorales, peuvent entraîner la libération de signaux de danger (ou DAMP, damageassociated molecular patterns) qui activent eux-mêmes différents acteurs du système immunitaire, notamment les cellules dendritiques: on parle de mort cellulaire immunogène [3].

L'homéostasie du système immunitaire est influencée par des facteurs intrinsèques mais également extrinsèques dépendant de l'environnement. L'étude de ces interactions permettrait d'optimiser l'efficacité des traitements utilisés dans le cancer. Le système nerveux central et le système neuroendocrinien peuvent également influencer le système immunitaire. En effet, l'expression de récepteurs de neuropeptides par les cellules immunitaires ainsi que celle de récepteurs de cytokines dans le cerveau illustrent bien cette communication bi-directionnelle. Les hormones sexuelles, certaines hormones du métabolisme, les peptides opioïdes sont autant de facteurs connus pour réguler le système immunitaire. Parmi toutes ces substances biologiques, les médiateurs du stress psychique ont un impact non négligeable qui semble délétère pour le système immunitaire [4]. Une récente méta-analyse montre que le stress psychique pourrait constituer un facteur de risque dans l'apparition des cancers [5]. Pourtant les mécanismes biologiques et immunologiques impliqués ne sont pas clairement définis. Ainsi, l'évaluation chez l'homme des effets de l'anxiété sur les réponses immunitaires anti-tumorales induites par différentes thérapies anticancéreuses et l'identification de moyens permettant de maîtriser ces effets est une approche innovante et encourageante. Dans une étude récente, Yang et al. ont exploré dans des modèles animaux l'impact de l'anxiété et de la variation de ses médiateurs sur la réponse immunitaire anti-tumorale [6]. 


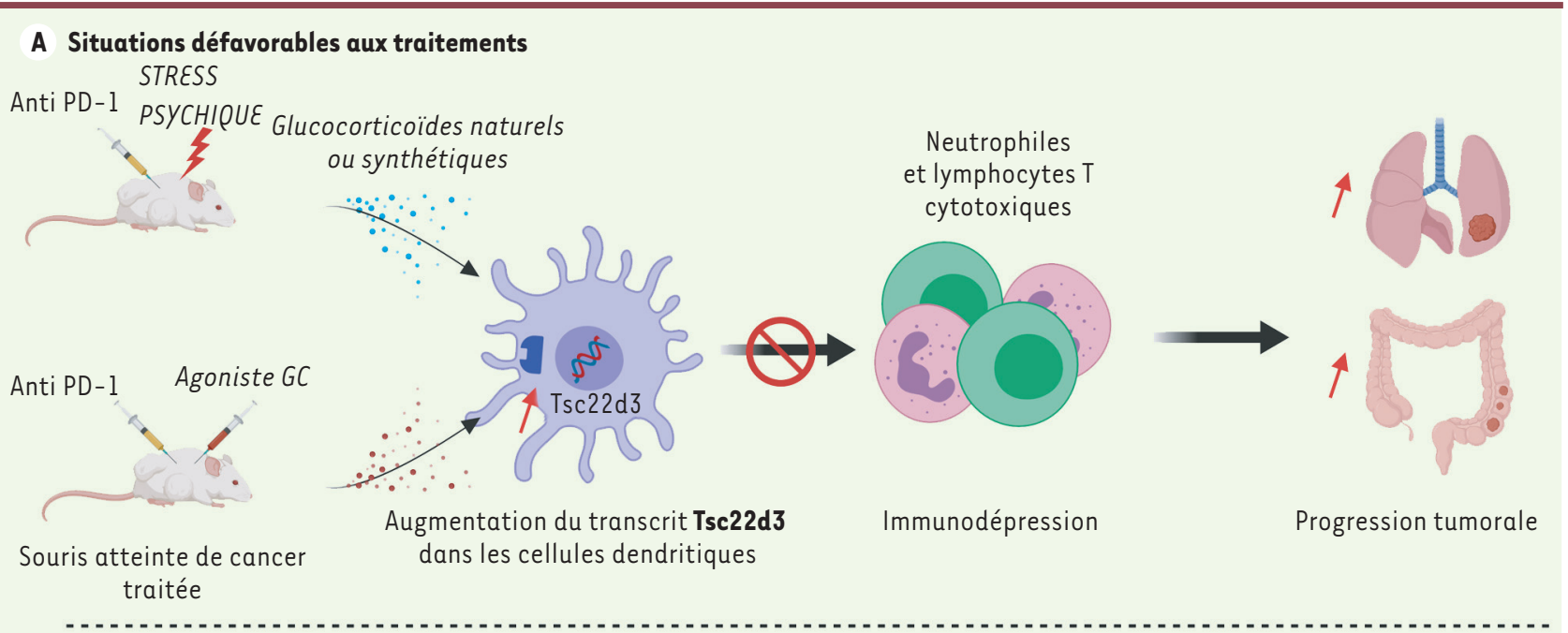

B Situations favorables aux traitements Anti PD-1

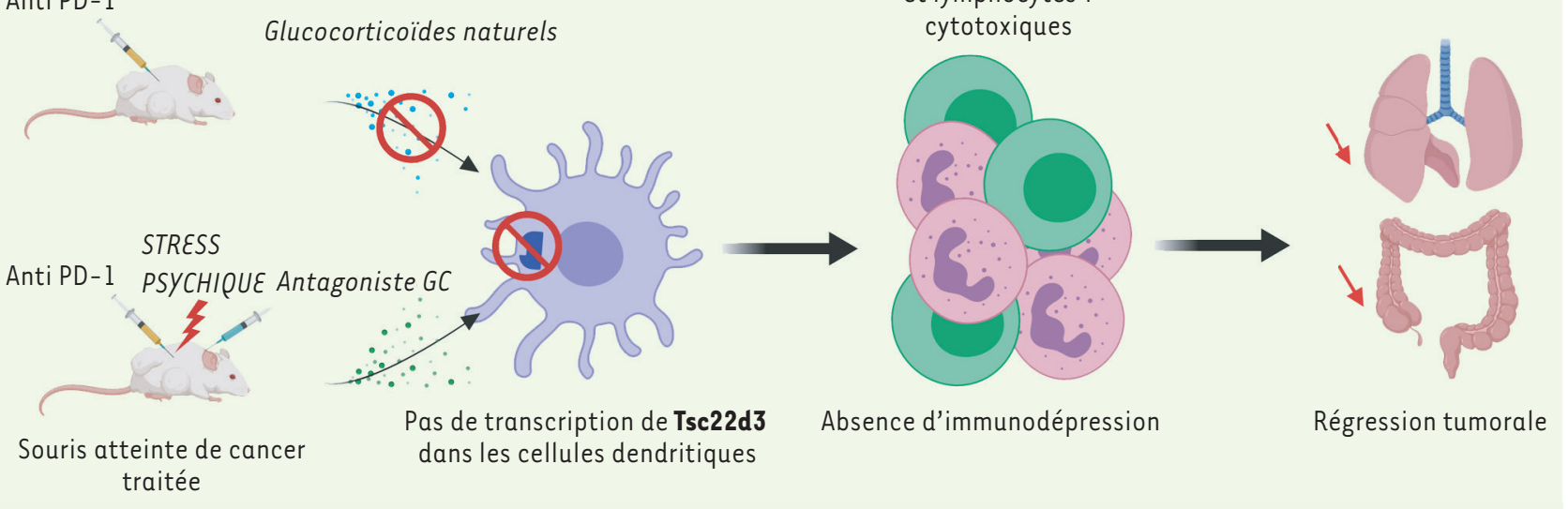

Figure 1. Effets du stress psychique sur la réponse anti-PD1. Impact de la surexpression du transcrit Tsc22d3 sur la réponse immunitaire antitumorale. GC : glucocorticoïdes

Le stress induit une immunodépression favorable à la progression tumorale et défavorable à l'efficacité des traitements anticancéreux

Dans cette étude, la confrontation d'une souris agressive avec une souris normale entraîne chez cette dernière un stress chronique de «défaite sociale » conduisant à un état d'anxiété ou de dépression. Ce conditionnement semble provoquer un état favorable à la progression tumorale dans les modèles étudiés (fibrosarcome, cancer du poumon non à petites cellules et du côlon) et diminuer l'efficacité de certaines chimiothérapies induisant une mort cellulaire immunogène, dont les sels de platine ou les anthracyclines. La réduction de la progression tumorale après traitement avec différentes immunothérapies, incluant l'utilisation d'un vaccin prophylactique (composé de cellules tumorales mourantes) ou des anticorps bloquants anti-PDl, est également diminuée chez les souris stressées par rapport aux souris contrôles.

Ces résultats sont corroborés par l'analyse du microenvironnement tumoral. $\varepsilon$ n effet, l'analyse de l'infiltrat tumoral chez les souris stressées révèle une plus faible infiltration des lymphoctyes T (LT) et des neutrophiles, deux populations cellulaires dont le rôle anti-tumoral est clairement établi [7]. Les auteurs ont également observé chez ces souris une augmentation des macrophages intratumoraux. En revanche, le nombre de cellules dendritiques dans le microenvironnement tumoral ne semble pas impacté par les conditions de stress. Les réponses Thl et Th2 chez ces souris sont diminuées alors qu'il n'y a pas de modification de la réponse régulatrice due aux lymphocytes T régulateurs (Treg). Ce déséquilibre peut donc être à l'origine d'une réponse immunosuppressive plus importante que la réponse effectrice anti-tumorale. De manière plus globale, les taux de 46 médiateurs plasmatiques de la réponse immunitaire incluant l'interféron gamma (IFN- $\gamma)$, qui est une cytokine pro-inflammatoire importante dans la lutte anticancéreuse, sont dimi- 
nués dans le plasma des souris stressées, témoignant que l'immunodépression est systémique et n'est pas restreinte au microenvironnement tumoral.

\section{L'augmentation du taux}

de glucocorticoïdes secondaire

à un stress psychique provoque

une immunodépression

Plusieurs médiateurs et neurotransmetteurs sont bien connus pour leur rôle dans la physiopathologie du stress. Les taux de glucocorticoïdes ${ }^{l}$ augmentent lors d'un stress chronique; à l'inverse, les taux de sérotonine (hormone impliquée dans la régulation des humeurs et du sentiment de bonheur) et de noradrénaline sont abaissés en situation de stress, chez l'homme et chez la souris. Pour autant, Yang et al. ont montré que seule l'inhibition des récepteurs des glucocorticoïdes chez les souris stressées (par injection d'un antagoniste) permet de rétablir la réponse immunitaire anti-tumorale ainsi que l'efficacité des immunothérapies et des chimiothérapies chez les souris stressées. De plus, l'utilisation d'analogue des glucocorticoïdes chez des souris non stressées réduit plusieurs fonctions immunitaires de manière similaire au stress décrit précédemment, et semble avoir un effet négatif dans la survie de souris traitées par différentes thérapies anticancéreuses. L'effet immunosuppresseur du stress psychique semble donc spécifiquement lié aux voies de signalisation activées par les glucocorticoïdes.

\section{Tsc22d3 : un facteur de transcription} responsable de l'immunodépression induite par le stress et les glucocorticoïdes

Parallèlement, la défaite sociale modifie également la présentation anti-

\footnotetext{
1 La cortisone et le cortisol sont les deux hormones glucocorticoïdes endogènes humaines, produites majoritairement par la zone fasciculée de la glande surrénale. La cortisone est le précurseur métabolique du cortisol, forme active de l'hormone. Le récepteur des glucocorticoïdes (naturels ou synthétiques), appelé $\mathrm{NR3Cl}$, est un récepteur nucléaire. La fixation du cortisol sur son récepteur entraîne sa translocation dans le noyau et sa fixation sur des promoteurs de gènes cibles.
}

génique, mécanisme immunologique impliquant en particulier les cellules dendritiques et nécessaire à l'initiation d'une réponse immunitaire adaptative. Des analyses transcriptomiques par RNAseq ont révélé une signature génétique immunosuppressive dans les cellules dendritiques (CD) infiltrant la tumeur des souris stressées avec notamment une surexpression de Tsc22d3, un facteur de transcription inductible par les glucocorticoïdes qui a un rôle anti-inflammatoire et immunosuppresseur. Cette surexpression est perdue lorsque les souris sont traitées avec des antagonistes des récepteurs des glucocorticoïdes, confirmant que la surexpression de Tsc22d3 est bien régulée par ces derniers. Dans un modèle de souris ayant subi un stress et dans lesquelles Tsc22D3 est absent, la réponse immunitaire est potentialisée : on observe une meilleure efficacité des anti-PDl, une ré-expression d'IFN- $\gamma$, une meilleure capacité de présentation antigénique et une plus grande infiltration par des LT, ainsi qu'une meilleure efficacité des immunothérapies ciblant PD-1.

Remarquablement, une augmentation, dans les cellules du sang périphérique et dans les CD intra-tumorales, de l'expression du facteur de transcription Tsc22d3 a également été observée chez des patients atteints de cancers digestifs (œsophage, estomac, côlon, rectum et pancréas), de cancer du poumon, ou de mélanome. Cette augmentation est corrélée à un état psychique négatif, évalué par le questionnaire POMS (Profile of Mood State) [8]. De plus, une forte expression de Tsc22d3 a été corrélée avec un mauvais pronostic pour la survie des patients dans de nombreux types de cancer, incluant le cancer du poumon et les carcinomes gastriques et colo-rectaux. On peut donc penser que les résultats découverts chez la souris pourraient favoriser la mise en place de stratégies thérapeutiques exploitables chez l'homme.

\section{Conclusions}

Ces travaux, particulièrement précurseurs dans leur approche et leurs conclusions, revêtent une importance particulière pour l'amélioration de la prise en charge des patients cancéreux. Au-delà du bien être mental des patients, une prise en charge psychologique de type psychothérapie ou par des moyens pharmacologiques pourrait donc avoir un effet direct sur l'efficacité des traitements en diminuant l'impact immunosuppresseur du stress. Ces approches ont d'ailleurs été évaluées dans des modèles de lymphomes avec des résultats encourageants [9]. Ces résultats posent également la question de la pertinence des glucocorticoïdes comme traitement adjuvant dans certains cancers non hématologiques. En effet, les glucocorticoïdes sont classiquement utilisés en complément des chimiothérapies pour réduire les douleurs et les vomissements [10]. Les données exposées ici laissent penser que la prise en charge de ces effets secondaires par des glucocorticoïdes pourrait avoir un réel impact négatif sur le traitement du cancer. Par ailleurs, il pourrait être intéressant d'évaluer l'effet chez des patients atteints de cancer de certains antagonistes des récepteurs des glucocorticoïdes déjà validés dans la maladie de Cushing (maladie caractérisée par un hypercortisolisme chronique). Bloquer ce récepteur sur les cellules dendritiques empêcherait les effets immunosuppresseurs des glucocorticoïdes. Cependant, les glucocorticoïdes étant des hormones pléiotropiques, bloquer leurs récepteurs pourrait conduire à des effets secondaires non négligeables. Enfin, pour identifier de nouvelles cibles thérapeutiques, le transcrit Tsc $22 \mathrm{~d} 3$ peut être envisagé comme un point de contrôle immunitaire (immune checkpoint) et son ciblage (inactivation ou élimination) pourrait améliorer l'immunosurveillance anticancéreuse et la réponse aux traitements. $\diamond$ 
Don't worry, be healthy!

How psychologic stress can drop down the efficacy of cancer therapy

\section{LIENS D'INTÉRÊT}

Les auteurs déclarent n'avoir aucun lien d'intérêt concernant les données publiées dans cet article.

\section{RéFÉRENCES}

1. Brunet JF, Denizot F, Luciani MF, et al. A new member of the immunoglobulin superfamily-CTLA-4. Nature 1987 ; 328: 267-70.

2. Hodi FS, O'Day SJ, McDermott DF, et al.. Improved survival with ipilimumab in patients with metastatic melanoma. $N$ Engl J Med 2010; 363 : 711-23.
3. Kroemer G, Galluzzi L, Kepp 0, Zitvogel L. Immunogenic cell death in cancer therapy. Annu Rev Immunol 2012 ; 31: 51-72.

4. Taub $D$. Neuroendocrine interactions in the immune system. Cell Immunol 2008 ; 252 : 1-6.

5. Batty GD, Russ TC, Stamatakis $\varepsilon$, Kivimäki M. Psychological distress in relation to site specific cancer mortality: pooling of unpublished data from 16 prospective cohort studies. BMJ 2017 ; 356 : j108.

6. Yang H, Xia L, Chien J, et al. Stress-glucocorticoidTSC22D3 axis compromises therapy-induced antitumor immunity. Nat Med 2019 ; 25 : 1428-41.

7. Schreiber RD, Old LJ, Smyth MJ. Cancer immunoediting: integrating immunity's roles in cancer suppression and promotion. Science 2011; $331: 1565-70$.

8. Baker F, Denniston M, Zabora J, et al. A POMS short form for cancer patients: psychometric and structural evaluation. Psychooncology $2002 ; 11: 273-81$.
9. Di Rosso ME, Sterle HA, Cremaschi GA, Genaro AM. Beneficial effect of fluoxetine and sertraline on chronic stress-induced tumor growth and cell dissemination in a mouse model of lymphoma: crucial role of antitumor immunity. Front Immunol $2018 ; 9: 1341$.

10. Pufall MA. Glucocorticoids and cancer. Adv Exp Med Biol $2015 ; 872$ : 315-33.

11. Teillaud JL. L'immunothérapie des cancers couronnée avec l'attribution du prix Nobel de Physiologie ou Médecine à James Allison et Tasuku Honjo. Med Sci (Paris) $2019 ; 35: 365-6$.

12. Fridman WH, Teillaud JL. De la traversée du désert au prix Nobel de Physiologie ou Médecine décerné à James Allison et Tasuku Honjo : la longue marche de l'immunothérapie des cancers. Entretien avec Wolf Hervé Fridman. Med Sci (Paris) 2019; $35: 367-73$. 